\title{
Stress, strain, and bulk microstructure in a cohesive powder
}

\author{
R. Andersson, ${ }^{1, *}$ W. G. Bouwman, ${ }^{1}$ S. Luding, ${ }^{2}$ and I. M. de Schepper ${ }^{1}$ \\ ${ }^{1}$ Radiation, Radionuclides \& Reactors, Delft University of Technology, Mekelweg 15, 2629JB Delft, The Netherlands \\ ${ }^{2}$ Multi Scale Mechanics, Department of Mechanical Engineering, University of Twente, P.O. Box 217, \\ 7500AE Enschede, The Netherlands
}

(Received 24 January 2008; published 9 May 2008)

\begin{abstract}
Spin-echo small-angle neutron scattering is able to characterize powders in terms of their density-density correlation function. Here we present a microstructural study on a fine cohesive powder undergoing uniaxial compression. As a function of compression, we measure the autocorrelation function of the density distribution. From these measurements we quantify the typical sizes of the heterogeneities as well as the fractal nature of the powder packing. The fractal dimension increases with increasing stress, creating a more space-filling structure with rougher phase boundaries. The microscopic stress-strain relation showed the same nonlinear behavior as the macroscopic relation. In this way it was possible to link the macroscopic mechanical response with the evolution of microstructure inside the bulk of the cohesive powder. The total macroscopic compressive strain is in agreement with a corresponding decrease in microstructural length scales.
\end{abstract}

DOI: 10.1103/PhysRevE.77.051303

PACS number(s): 81.05.Rm, 61.43.Gt, 61.43.Hv

\section{INTRODUCTION}

Rather than being driven by the hard-core exclusion between grains, the structure of cohesive powders is mainly determined by the van der Waals attraction among small primary particles $(<100 \mu \mathrm{m})$. As a result of these adhesive forces, and the irrelevancy of body forces, very loosely packed and fluffy structures are seen to form in these materials. The structures lead to process-related issues relevant to industry in a sense that mass flows become unstable [1]. An increased demand for particulate materials on the nanoscale makes the understanding of this type of granular material important.

To precisely understand the macroscopic behavior of powders, the computer modeling and theory buildup needs the support of experiments on realistic samples. More specifically, there is a need for experiments that can quantify powders in terms of their microstructure. Experiments are needed that can look inside the "fluffy" structure of cohesive powders so that more quantitative statements can be made.

Extracting information from the bulk of powders and granular materials is difficult. The opacity and the wide range of sizes present in real materials render most optical and conventional wave diffraction techniques more or less useless. The opacity can be overcome by using penetrating radiation such as $\mathrm{x}$ rays in $\mathrm{x}$-ray tomography [2-4] or radio waves in magnetic resonance imaging $[5,6]$.

Understanding macroscopic mechanical behavior can be obtained by investigating the changes in crystallographic microscopic parameters [7]. Following the microscopic evolution by using penetrating radiation makes it possible to understand the macroscopic development from the microscopic point of view. Methods for studying the mechanical properties at the grain level of granular packings exist [8], and it is usually the buildup of force chains that is addressed. Neutron diffraction has been used to study nonlinear stress-strain behavior in granular materials [9].

\footnotetext{
*r.a.andersson@tudelft.nl
}

In order to access the bulk of more realistic materials it becomes necessary to use penetrating radiation and a technique that has enough resolution to analyze the small-angle scattered radiation. Spin-echo small-angle neutron scattering (SESANS) is a high-resolution technique which measures the autocorrelation function of the sample density distribution in real space [10]. SESANS can be used to probe the structure across three orders of magnitude ranging from 30 $\mathrm{nm}$ up to $20 \mu \mathrm{m}$, making it applicable to fine cohesive powders [11], colloidal systems [12], and dairy products [13], just to name a few.

The autocorrelation function of the density distribution $\gamma(r)$ can be used to characterize the microstructure of materials. This function is measured in a small-angle scattering experiment as its Fourier transform (the so-called structure or form factor). SESANS measures $\gamma(r)$ via its projection along the neutron beam path, making SESANS a real-space technique. The typical size, packing fraction, any anisotropy, scale invariance, ordering, etc. of the sample heterogeneities are examples of extractable sample properties.

A stress-strain measurement on a fine cohesive silica powder together with consecutive SESANS measurements has been performed and is reported in this paper. In the analysis, the cohesive powder is considered as being a self-affine random two-phase material. We use a model function for the density-density correlation function containing a typical size $a$ of the structure and the so-called Hurst exponent $H$, related to the fractal dimension of the structure $[14,15]$. The Hurst exponent depends on the phase boundary roughness, and the higher the degree of "surface roughness" the lower is the Hurst exponent.

From the initial decay of the measured SESANS curve it is possible to extract the unknown grain density and consequently the grain packing fraction. We find that the typical size of the inhomogeneities decays in a nonlinear way with increasing compressive strain, and the microscopic stressstrain relationship shows the same exponential behavior as the macroscopic stress-strain curve. The Hurst exponent is seen to decrease with increasing stress and strain, showing that a more space-filling structure with rougher interfaces is 
being formed as a function of compression. Altogether, the study links the nonlinear compressive stress-strain relationship to the evolution of microstructural parameters.

\section{MICROSTRUCTURE AND SESANS}

\section{A. Density distribution and its correlation function}

The density distribution $\rho(r)$ in a heterogeneous twophase sample is expected to fluctuate around its mean value. These fluctuations might be characterized by a typical size, anisotropy, being fractal or self-affine, regular (crystalline), random, and so on. Taken all together, this is what we call the structure of a material. The structure of two-phase systems such as a powder material can be analyzed in terms of the autocorrelation function of its density distribution:

$$
C(r)=\langle\Delta \rho(0) \Delta \rho(r)\rangle,
$$

where the mean $\bar{\rho}$ has been subtracted $[\Delta \rho(r)=\rho(r)-\bar{\rho}]$. The mean square fluctuation is

$$
C(0)=\Delta \rho_{0} \phi_{1} \phi_{2},
$$

where $\phi_{1}+\phi_{2}=1$ are the packing fractions of the two phases and $\Delta \rho_{0}=\rho_{1}-\rho_{2}$ is the solid density difference between the two phases. The normalized (dimensionless) correlation function is

$$
\gamma(r)=\frac{C(r)}{C(0)} .
$$

The correlation function can be expressed in terms of the pair correlation function $g_{2}(r)[16] . g_{2}(r)$ gives the probability of finding a particle center of mass at a distance $r$ away from the origin, given that there is a particle at that origin. This function is especially useful for the study of monodisperse sphere packings. For spheres, the correlation function becomes

$$
\gamma(r)=\gamma_{0}(r)+2 \int_{0}^{\infty} \gamma_{D}(r, c) g_{2}(c) c^{2} d c,
$$

where $\gamma_{D}(r, c)$ is the autocorrelation of a pair of spheres separated by $c$ [17] and $\gamma_{0}(r)$ is the autocorrelation function of a sphere.

The projection of $\gamma(r)$ is given by

$$
G(z)=\frac{2}{\xi} \int_{z}^{\infty} \frac{r \gamma(r)}{\sqrt{r^{2}-z^{2}}} d r
$$

and in Cartesian coordinates by

$$
G(z)=\frac{1}{\xi} \int_{-\infty}^{\infty} \gamma(x, 0, z) d x .
$$

The projection is made dimensionless with the correlation length of the density distribution [18],

$$
\xi=\int_{-\infty}^{\infty} \gamma(r) d r
$$

so that $G(0)=1$.

\section{B. Spin-echo small-angle neutron scattering}

Spin-echo small-angle neutron scattering is based on the Larmor precession of neutrons in parallelogram-shaped magnetic field regions $[19,20]$. In SESANS, the polarization of a neutron beam is measured, after transmission through a sample, as a function of the so-called spin-echo length (30 $\mathrm{nm}<z<20 \mu \mathrm{m}$, set by the instrument user). The spinecho length is a real-space parameter representing the size at which the correlations are measured (in the $z$ direction of the laboratory-coordinate system). In SESANS, $G(z)$ is measured through the transmission of polarization, normalized by experimental effects, as a function of $z$,

$$
P(z)=e^{\Sigma_{t}[G(z)-1]},
$$

where

$$
\Sigma_{t}=t \lambda^{2} \Delta \rho_{0}^{2} \phi_{1} \phi_{2} \xi .
$$

Here $t$ is the sample thickness, $\lambda$ the neutron wavelength, and $\Delta \rho_{0}$ the neutron-scattering-length density difference in the sample (i.e., the contrast between the two phases in the sample). Note that Eq. (8) takes into account the effects of multiple scattering [21].

The correlation length $\xi$ is measured along the neutron beam axis of a SESANS experiment, which is perpendicular to the $z$ direction. $\xi$ is a measure of the width of the distribution $\gamma(r)$, which is in principle a measure of the size of the inhomogeneities in the sample (for a sphere the correlation length is $3 / 4$ of the sphere diameter). It can be interpreted as the mean free path of a neutron in the sample.

At large $z$ (above the largest size describing the heterogeneities) one expects no more correlations $[G(\infty)=0]$; this gives the so-called saturation level of the polarization:

$$
P(\infty)=e^{-\Sigma_{t}} .
$$

Thus, the logarithm of the polarization at saturation is proportional to the correlation length $\xi$ of the sample inhomogeneities.

\section{Correlation function of a random two-phase system}

A cohesive powder is a particular case of a two-phase system that is heterogeneous at (most likely) many scales. The attractive forces between grains allow for the buildup of connected networks and aggregates of low coordination number, resulting in large voids of air pockets and, in essence, low densities. In the end we have in mind a very porous material carrying a low packing fraction of grains that will be far from a random close packing of hard spheres, or any other ordered density distribution.

For a perfectly random heterogeneous material made up of three-dimensional (3D) solids bounded by smooth 2D surfaces, the density-density correlation function can be described by the so-called Debye-Andersson-Bueche (DAB) formalism [22-24]:

$$
\gamma(r)=e^{-r / a},
$$

where $a$ is a measure of the typical size of the heterogeneities. This function can be seen as a special case of the more 

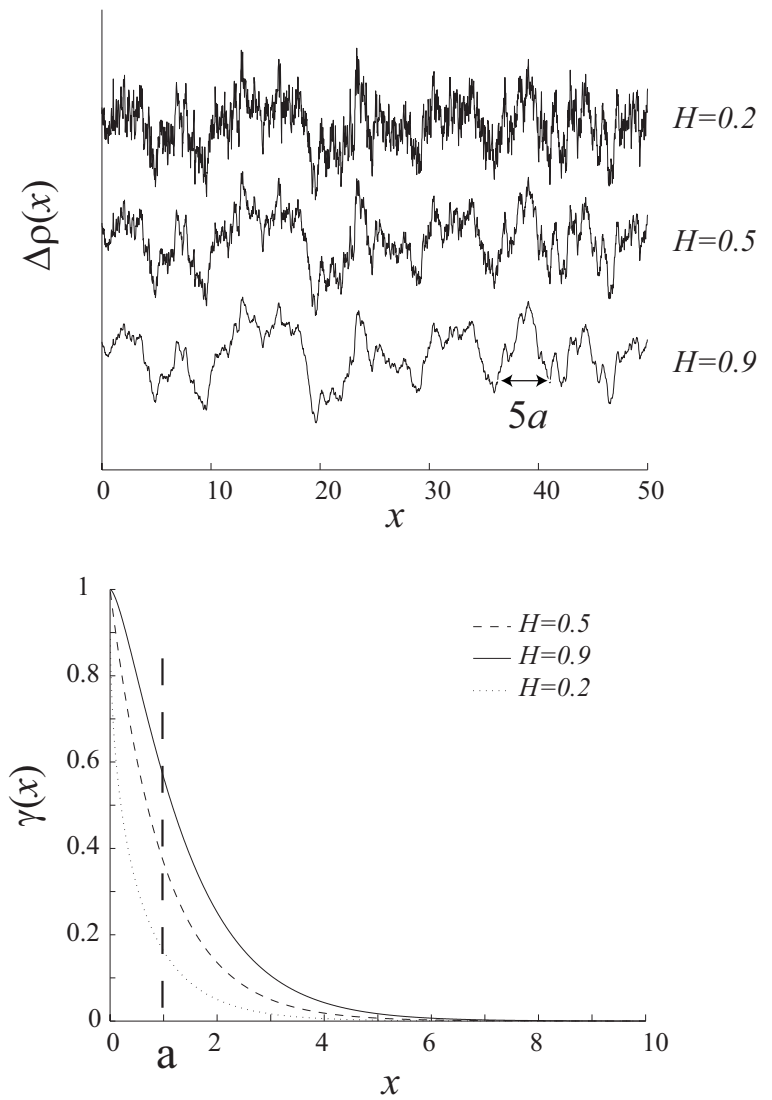

FIG. 1. Top: Examples of reconstructed 1D density distributions $\rho(x)$ based on the correlation function Eq. (12). Bottom: Corresponding correlation functions. The characteristic size is here $a=1$.

general von Karman correlation function [14,25]

$$
\gamma(r)=\frac{2}{\Gamma(H)}\left(\frac{r}{2 a}\right)^{H} K_{H}\left(\frac{r}{a}\right),
$$

where $K_{H}$ is the second-order modified Bessel function and $\Gamma$ is the Gamma function. For $H=1 / 2$ this simplifies to the DAB formula in Eq. (11).

The so-called Hurst exponent $0<H<1$ is related to the dimensionality of the structure. The limits $H=0$ and 1 correspond to a space-filling and a smooth Euclidian distribution, respectively. In this context it is related to the interface roughness between the two phases making up the material. One usually discusses two domains, $H>1 / 2$ where the distribution is persistent and characterized by a certain degree of memory and longer-ranged correlations; and the domain $H<1 / 2$ describing an antipersistent distribution, governed by shorter-ranged correlations (i.e., rougher).

The Hurst exponent has been used to analyze the structure of shear bands [26] as well as for the study of percolation in porous materials [27] and in fracture profiles [28].

A 1D reconstruction of density distributions for various Hurst exponents can be seen in Fig. 1. This calculation is done in the wave number domain where a uniform deviate (white noise) is filtered with a spectral filter. The inverse Fourier transformation of the filtered white noise yields a real-space representation of the distribution. The spectral fil- ter is the square root of the Fourier transformation of the autocorrelation function, in this case Eq. (15) (see also [14]).

The projection of Eq. (12) is found by insertion in Eq. (5), which leads to

$$
G(z)=\frac{2}{\Gamma(H+1 / 2)}\left(\frac{z}{2 a}\right)^{H+1 / 2} K_{H+1 / 2}\left(\frac{z}{a}\right) .
$$

The corresponding correlation length for this density distribution will be

$$
\xi=\frac{2 \sqrt{\pi} a \Gamma(H+1 / 2)}{\Gamma(H)} .
$$

In conventional small-angle neutron scattering one measures the Fourier transform of Eq. (12), which yields the normalized intensities as a function of wave number,

$$
I(q)=\frac{1}{\left[1+(q a)^{2}\right]^{3 / 2+H}} .
$$

\section{EXPERIMENTS AND SAMPLE PROPERTIES}

A powder sample was kindly provided by Degussa [32]. The product is called Sipernat-310; it is a synthetic precipitated silica used in coatings, cosmetics, cements, rubbers, as filler, etc. It is a typical cohesive powder containing fine grains around $5 \mu \mathrm{m}$ in diameter. The sample was used without any further treatment, in ambient conditions.

We have used the SESANS setup at the Reactor Institute Delft (Delft University of Technology in the Netherlands) to perform the measurements. The instrument contains two parallelogram-shaped magnetic field regions with opposite magnetic induction directions (otherwise identical). The sample is positioned between the field regions. Any neutron scattering between the two fields will break the symmetry of the setup and cause the beam to depolarize. The strength of the field defines the so-called spin-echo length $z$, which is perpendicular to the beam direction and pointing in the direction of gravity. The polarization of the neutron beam is measured as a function of $z$. The beam is nearly monochromatic with a wavelength of $0.21 \mathrm{~nm}$, having a cross section at the sample position around $1 \mathrm{~cm}^{2}$.

A simple uniaxial load cell was used in order to measure the stress versus strain function of the powder (see Fig. 2). Strain is here defined as being the relative decrease in thickness of the sample,

$$
\delta t=1-\frac{t}{t_{0}},
$$

where $t$ is the thickness after compressive straining and $t_{0}$ is the initial sample thickness. The stress and strain tester contains a cylindrical cavity with a movable hollow plunger that achieves the compression inside the cavity. The plunger was moved by a separate screwing action (without rotating the plunger) until a desired incremental strain was reached. We used nine increments of $0.25 \mathrm{~mm}$ with an initial powder height of $6.5 \mathrm{~mm}$. The cylindrical cavity and the plunger are sealed off with aluminum windows (aluminum is virtually 


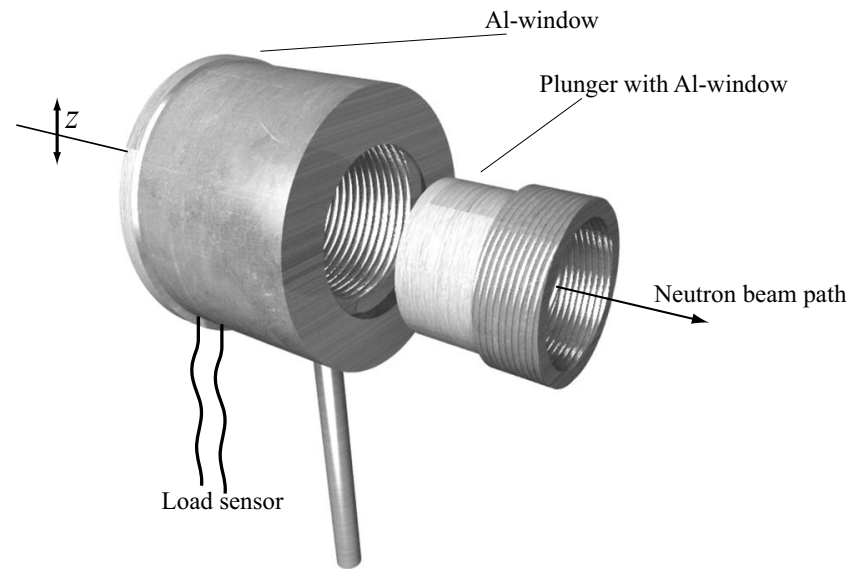

FIG. 2. Load cell used in the stress-strain measurements on the cohesive powder. The initial height of the powder packing was 6.5 $\mathrm{mm}$ and it was subsequently strained by nine increments of 0.25 $\mathrm{mm}$. The stress was measured using Flexiforce load sensors situated at the first $\mathrm{Al}$ window.

transparent to neutrons). The absolute stress was measured with Flexiforce load sensors provided by Tekscan [33].

The initial powder packing is a very soft, low-density material and easily compacted (weakly aggregated). As seen in the stress-strain curve, which shows an exponential variation of stress as a function of strain in the probed interval (Fig. 3). The goal of this study is to understand this nonlinear stress-strain relationship through the bulk microstructure at each point of the stress-strain curve.

\section{RESULTS}

\section{A. First interpretation}

When analyzing the measurements it is often useful to interpret what we simply see by eye. In Fig. 4 the polariza- tion is plotted as a function of $z$. In total, ten measurements at ten different strain levels were carried out. The figure shows only four of the ten measurements for the sake of clarity.

Increasing the strain moves the saturation polarization upward; thus the upper curves correspond to higher stress and strain than the lower ones. Increasing the strain makes the powder packing denser $(\phi)$ and thinner $(t)$, both contributing to less scattering and higher polarization saturation levels.

The second microstructural parameter contributing to the end level is the correlation length $\xi$. The rearrangement of particles into a denser state must decrease the size of the heterogeneities, giving rise to a lower correlation length with increasing strain.

At saturation we are able to read off the largest correlating size of the microstructure on the horizontal axis. The measurement saturates around $5 \mu \mathrm{m}$, which is the size of grains making up the powder. Thus, no correlations are seen beyond the size of a grain.

The stress versus strain curve can be seen in Fig. 3. The curve shows that when the logarithm of the stress versus strain is plotted a linear relationship is obtained. The powder in its native state is a very soft powder with low density stabilized by adhesive forces in the form of capillary and van der Waals forces. The hard-core exclusion between the silica grains finally governs the interaction between grains, as can be seen in the divergent behavior of the applied stress for lower characteristic sizes (see the upper left plot in Fig. 6).

\section{B. Linear initial slopes}

The powder is composed of grains having an unknown solid density. The grain density will be lower as compared with the solid density of pure silica $\left(\rho_{\mathrm{SiO}_{2}}=2.2 \mathrm{~g} / \mathrm{cm}^{3}\right)$. From the grain density it is then possible to calculate the grain

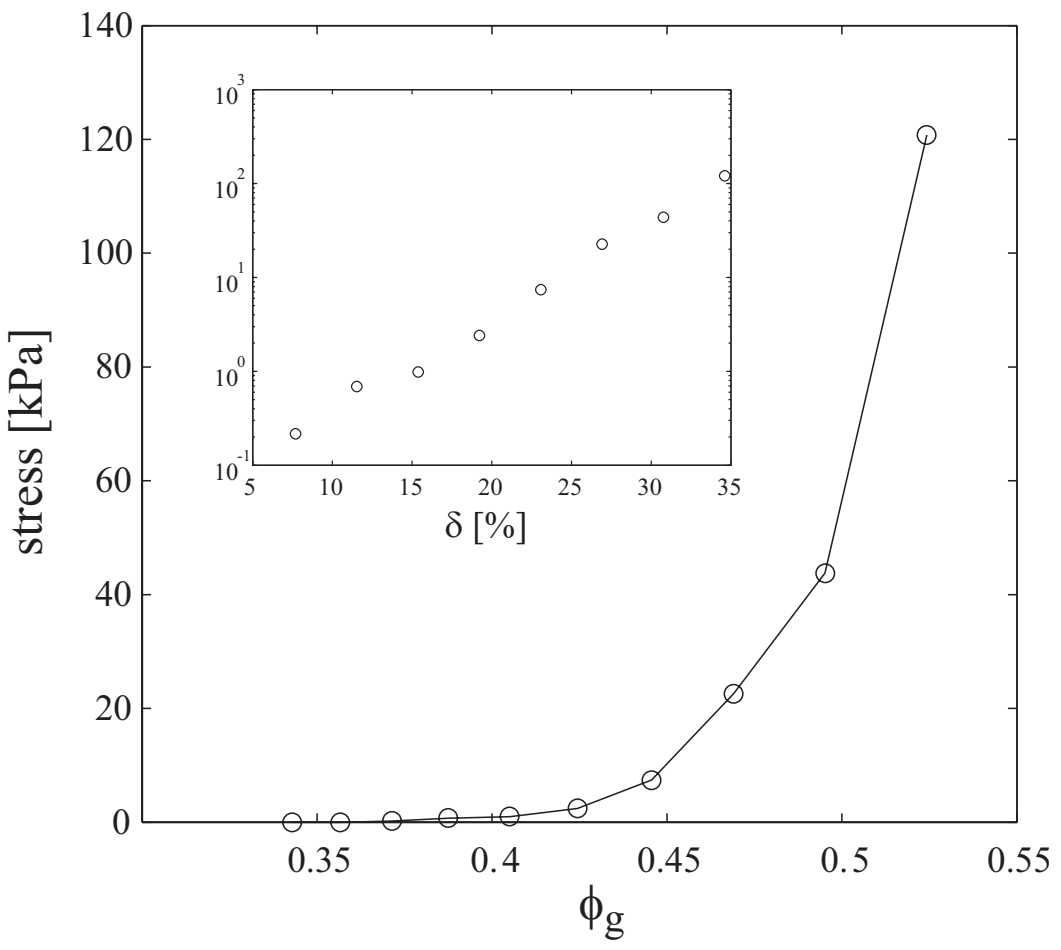

FIG. 3. Stress versus grain packing fraction $\phi_{\text {grain }}$ relationship for the Sipernat-310 powder. The inset shows a linear relationship between the logarithm of the stress versus strain $\delta$. Note that the first two points yielded no measurable stress on the Flexiforce load sensors. 


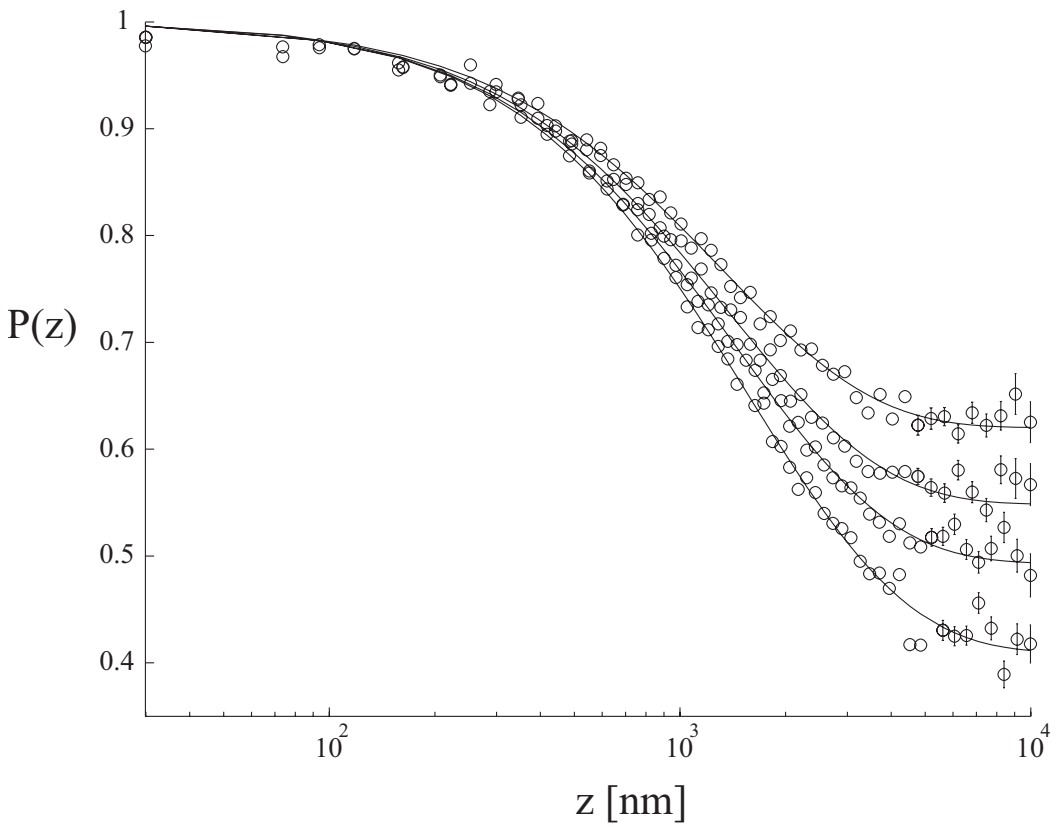

FIG. 4. Polarization plotted as a function of $z$. From top to bottom these measurements correspond to a uniaxial stress (strain) of $120 \mathrm{kPa}$ (35\%), $7.4 \mathrm{kPa}(23 \%), 1 \mathrm{kPa}(15 \%)$, and $0 \mathrm{kPa}$ $(3.8 \%)$. The full curves are fitted according to Eqs. (13), (14), and (8). Unless shown, the error falls within the marker symbol.

packing fraction $\phi_{\text {grain }}$ rather than the skeleton packing fraction $\phi_{\mathrm{sk}}$, which is calculated from the solid silica density. To summarize,

$$
\phi_{\mathrm{sk}}=\frac{\rho_{\mathrm{sample}}}{\rho_{\mathrm{SiO}_{2}}}
$$

and

$$
\phi_{\text {grain }}=\frac{\rho_{\text {sample }}}{\rho_{\text {grain }}},
$$

where $\rho_{\text {sample }}$ is the density of the powder packing and $\rho_{\mathrm{SiO}_{2}}$ is the solid density of pure silica.

The grain density and the grain packing fraction can be determined from the SESANS measurement by analyzing the initial slope of the $P(z)$ vs $z$ curves. The final amplitude of the polarization (saturation level) is given by Eq. (10) and is read at a spin-echo length $z$ related to $\xi$. Thus, the gradient of the initial part of $P(z)$ vs $z$ yields a quantity that depends only on the sample thickness, packing fraction $\phi$, and scattering length density $\Delta \rho_{0}$. The slope is in a sense independent of structural arrangement of the density,

$$
-\frac{d \ln (P)}{d z} \simeq t \lambda^{2} \Delta \rho_{0}^{2} \phi(1-\phi) .
$$

Thus, division of the values of the slopes by the sample thickness and the primary particle packing fraction $\phi_{\text {grain }}$ should yield a constant term for all experiments (see bottom right figure in Fig. 5), because straining the sample does not
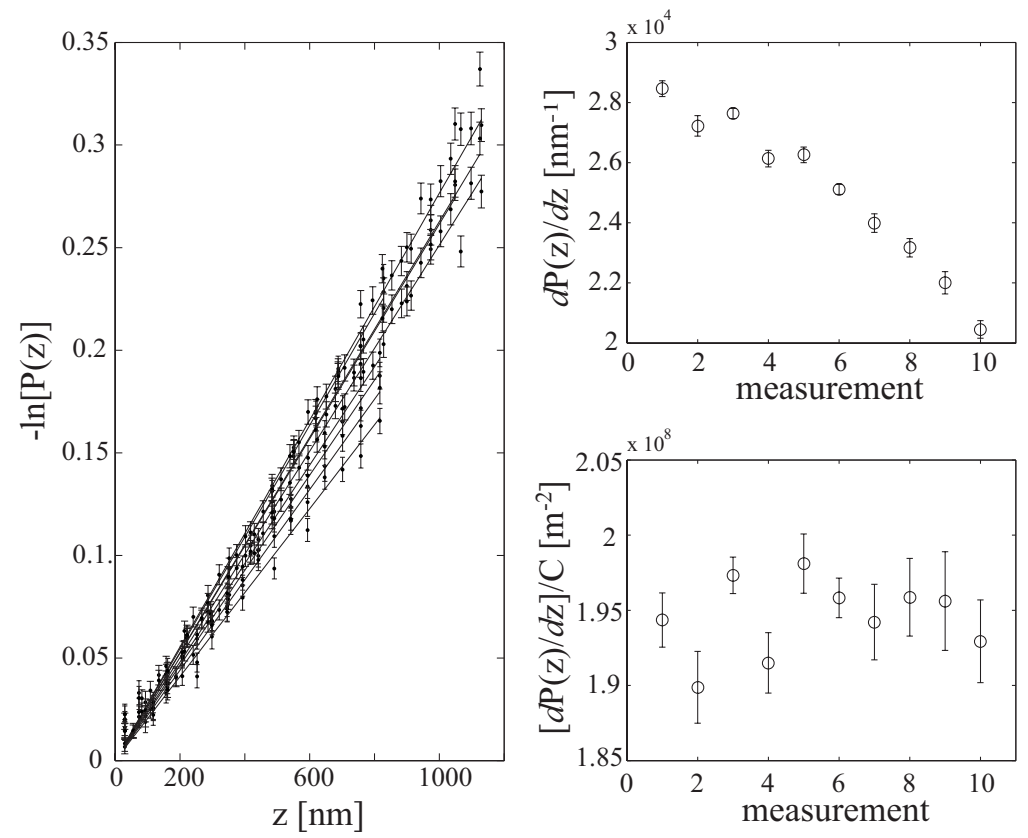

FIG. 5. Initial slopes of the polarization $d \ln (P) / d z$. The slopes are proportional to the grain packing fraction and the sample thickness multiplied by a constant [see Eq. (19)]. Dividing out the known thickness and the packing fraction $\left[C=\phi_{\text {grain }}\left(1-\phi_{\text {grain }}\right) t\right]$ should yield a constant term for all measurements as seen in the lower right figure. This analysis makes it possible to determine the solid grain density, yielding about $1.1 \mathrm{~g} / \mathrm{cm}^{3}$. 

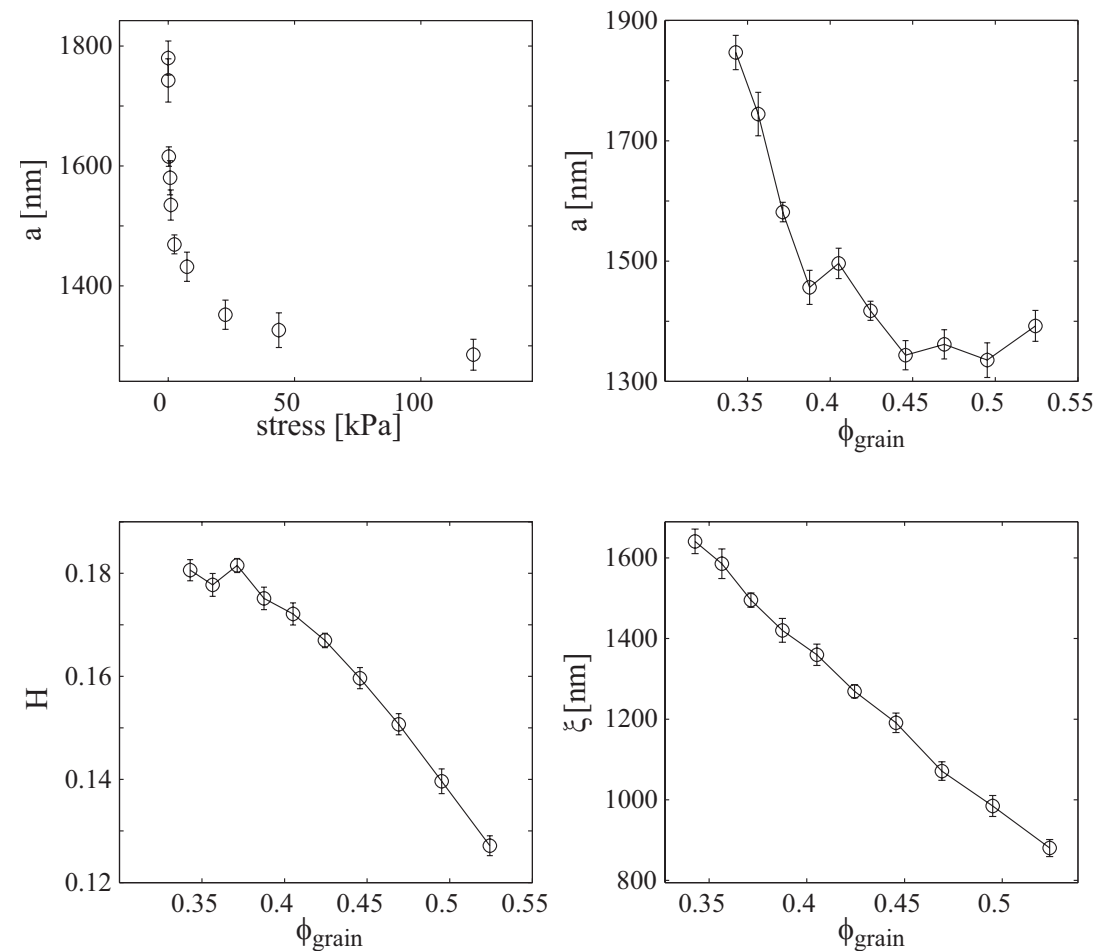

FIG. 6. Characteristic size $a$ and Hurst exponent $H$ obtained when applying the model Eq. (13) to the measurement. The model shows that the characteristic size as well as the Hurst exponent $H$ decrease as a function of compression (higher $\phi_{\text {grain }}$ ). The packing fractions are here expressed in terms of the grain packing fraction $\phi_{\text {grain }}$ defined in Eq. (18) change $\Delta \rho_{0}$ or $\lambda$. We find the grain packing fraction $\phi_{\text {grain }}$ by fitting until a nearly constant term is found for all measurements. The grain density can then be found by using Eq. (18).

This analysis gives a density of $\rho_{\text {grain }}=1.1 \mathrm{~g} / \mathrm{cm}^{3}$ and packing fractions ranging from $\phi_{\text {grain }}=0.34$ up to 0.53 for the highest strain.

\section{Curve shapes and their amplitudes}

For a more complete analysis of the SESANS experiment, we apply a model that describes an autocorrelation function of the density distribution. From that model we are able to calculate the corresponding projection as well as the expected polarization shapes and amplitudes.

The thickness $t$, packing fraction $\phi$, and neutron wavelength $\lambda$ are all known experimental parameters that contribute to the saturation level $P(\infty)$. The scattering length density $\Delta \rho_{0}$ is a constant parameter given by the chemical composition of the sample. Thus, in order for the model to be consistent, it has to yield a constant scattering length density for all ten measurements. In short, we have to find a model capable of describing $\gamma(r)$ as well as $\xi$ in such a way that it produces the saturation level and curve shapes observed in the measurement.

To model the data we use the von Karman correlation function introduced in Sec. II C, which describes a statistical self-affine density distribution according to Eqs. (12)-(14). The model explains the microstructure with two parameters, the Hurst exponent $H$ and a characteristic size $a$ of the density distribution. This yields, including experimental parameters, a scattering length density of about $1.1 \times 10^{14} \mathrm{~m}^{-2}$ for all samples.

The model parameters measured at each incremental strain are plotted in Fig. 6. The characteristic size rapidly decreases with increasing strain before it saturates for strains larger than $20 \%-25 \%$. In order for the powder to be compressed the larger inhomogeneities, clusters and voids, have to be broken and collapsed. This first stage occurs at the lower stress amplitudes and produces relatively large changes in $a$. When the larger and weaker heterogeneities have collapsed and produced a denser structure, any further densification is created by the rearrangement of primary grains. This latter stage is governed by higher stresses and smaller changes in the characteristic size of the heterogeneities. This "hard-core" behavior is illustrated when we plot the stress versus the characteristic size $a$ in Fig. 6. The stress diverges at around $1400 \mathrm{~nm}$ and no significant change is seen for higher stresses. The total microscopic strain for the experiment is given by

$$
\delta a_{\mathrm{tot}}=1-\frac{a_{\mathrm{end}}}{a_{0}}=1-\frac{1290}{1780} \simeq 28 \%,
$$

where $a_{\text {end }}$ is the characteristic size reached at the maximum applied strain and $a_{0}$ is the size at the beginning. This value is comparable with the final macroscopic strain, being about $35 \%$ (see Fig. 3).

The Hurst exponent decreases with increasing strain, consistent with the formation of a more space-filling structure as well as with an increase in the phase boundary roughness. The short-range structure becomes more irregular, creating more sliding contacts, which contributes to the nonlinear stress-strain behavior and in particular makes the structure more resistant toward straining.

The two microscopic strains are

$$
\delta a=1-\frac{a}{a_{0}}
$$




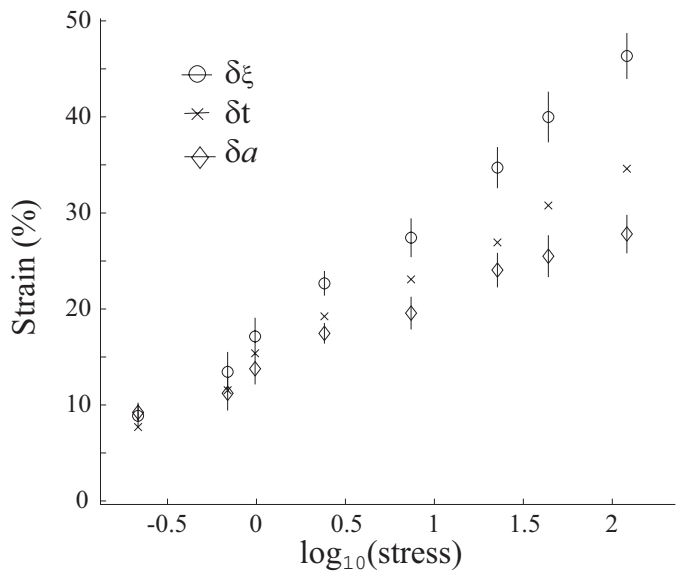

FIG. 7. Logarithm of the stress plotted as a function of microstructural strains $\delta a$ and $\delta \xi$ together with the macroscopic stressstrain relationship.

$$
\delta \xi=1-\frac{\xi}{\xi_{0}}
$$

The microscopic strain is plotted versus the logarithm of the stress in Fig. 7. This shows that the exponential stress-strain relationship measured macroscopically has its origin in a similar relationship at the microscopic level.

\section{DISCUSSION AND CONCLUSIONS}

A stress-strain measurement was conducted simultaneously with a bulk microstructural investigation by using spin-echo small-angle neutron scattering on a cohesive silica powder (Sipernat-310). The microstructure was characterized in terms of the autocorrelation function of the density distribution, and the experimental result was modeled using a correlation function describing a random density distribution. The correlation function characterizes the density distribution in terms of its typical size $a$ and a self-affine parameter called the Hurst exponent $H$, related to the short-range correlations, i.e., to the structure of the phase boundaries. The proposed correlation function proved excellent at describing our measured data.

The primary grain density was determined by analyzing the initial slopes of the SESANS measurements. This yields a density of about a factor of 2 smaller than the skeleton density of pure silica. The grain density can be used to calculate the packing fraction of grains contained in the sample. A divergence of the stress is reported at a packing fraction around 0.50

The fractal nature of the powder can be quantified in terms of the so-called Hurst exponent. When the powder is compressed, decreasing Hurst exponents are evidenced as a function of strain. A decreasing Hurst exponent implies that a more "disordered" density distribution is being formed, and we argue that such a structure can pack more efficiently, thus facilitating the compression of the powder. The decrease in the Hurst exponent also indicates the buildup of a rougher, more disordered, interface. Similar observations have been made in metals [29], shear zones in granular materials [26], and fracture studies [28]. The increase in interface roughness creates more sliding contacts and friction between grains, in essence acting against the action of compression, and contributes to the nonlinear stress-strain behavior observed in the macro- as well as in the microscopic domain.

Compression of the powder using relatively small stress levels breaks and collapses the larger voids and clusters that are stabilized by weaker van der Walls forces. This phenomenology is supported by the measurement in the sense that the microstructural length scale $a$ decreases quickly for relatively low stresses. Further densification can be achieved through the movement and rearrangement of hard primary grains. Such a structural rearrangement calls for larger stress levels. The measurement shows a sharp diverging stress for smaller characteristic sizes $a$, and we argue that a domain is reached where the structural rearrangement is governed by hard-core exclusions.

The total microscopic strain $\delta a_{\text {tot }}(28 \%)$ observed in the experiment is comparable to, but lower than, the value for the total macroscopic strain of the sample $\sim 35 \%$. When the microscopic stress-strain relationship is further analyzed it is evident that the macroscopic exponential behavior has its origin in a similar microscopic relationship.

It is well established that force networks and chains play an important role in the physics of granular matter [30]. In the measurements conducted here and elsewhere [31], no density correlations were observed beyond the size of a single grain. The measurements can lead to the conclusion that (if present) such networks do not create correlations in the density distribution.

As opposed to discussing the density distribution in terms of a sphere and its diameter, or any other shape of a grain and its size, we argue that parameters such as $a$ (the width of the autocorrelation function of the density distribution) and also $\xi$ (the correlation length of the distribution) represent more general and even well-defined descriptors of the material's microstructural size, especially when realistic materials are considered, materials that typically contain many different shapes having many different sizes.

An important connection between microstructure and macroscopic mechanical behavior of a powder has been made. The study was made possible by a unique neutron scattering investigation using SESANS. It was shown that the random media model, given by the von Karman correlation function, is excellent at describing the structure of a cohesive powder in a wide range of packing fractions, and the model parameters involved provide insight into the mechanics and microstructure of the compressed powder.

\section{ACKNOWLEDGMENTS}

We thank Chris Duif at the Reactor Institute Delft for valuable discussions and help with performing the experimental part of this work. This work is part of the research program of the "Stichting voor Fundamenteel Onderzoek der Materie (FOM)," which is financially supported by the "Nederlandse Organisatie voor Wetenschappelijk Onderzoek (NWO)." 
[1] J. Tomas, Granular Matter 6, 75 (2004).

[2] T. Aste, M. Saadatfar, and T. J. Senden, Phys. Rev. E 71, 061302 (2005).

[3] P. Richard, P. Philippe, F. Barbe, S. Bourlès, X. Thibault, and D. Bideau, Phys. Rev. E 68, 020301(R) (2003).

[4] G. T. Seidler, G. Martinez, L. H. Seeley, K. H. Kim, E. A. Behne, S. Zaranek, B. D. Chapman, S. M. Heald, and D. L. Brewe, Phys. Rev. E 62, 8175 (2000).

[5] A. Sederman, P. Alexander, and L. Gladden, Powder Technol. 117, 255 (2001).

[6] D. Mueth, G. Debregeas, G. Karczmar, P. Eng, S. Nagel, and H. Jaeger, Nature (London) 406, 385 (2000).

[7] V. Hauk, Structural and Residual Stress Analysis by Nondestructive Methods (Elsevier, Amsterdam, 1997).

[8] T. Majmudar and R. Behringer, Nature (London) 435, 1079 (2005).

[9] T. W. Darling, J. A. TenCate, D. W. Brown, B. Clausen, and S. C. Vogel, Geophys. Res. Lett. 31, L16604 (2004).

[10] W. G. Bouwman, O. Uca, S. V. Grigoriev, W. H. Kraan, J. Plomp, and M. T. Rekveldt, J. Appl. Phys. 74, S115 (2002).

[11] R. A. Andersson, W. G. Bouwman, and I. M. de Schepper, Powders and Grains (Balkema, Stuttgart, 2005), pp. 13-15.

[12] T. Krouglov, I. M. de Schepper, W. G. Bouwman, and M. T. Rekveldt, J. Appl. Crystallogr. 36, 117 (2003).

[13] H. Tromp and W. G. Bouwman, Food Hydrocolloids 21, 154 (2007).

[14] L. Klimes, Pure Appl. Geophys. 159, 1811 (2002).

[15] B. B. Mandelbrot, The Fractal Geometry of Nature (W.H. Freeman and Company, San Francisco, 1982).

[16] T. Kruglov, J. Appl. Crystallogr. 38, 721 (2005).

[17] O. Glatter, Acta Phys. Austriaca 52, 243 (1980).

[18] L. Feigin and D. Svergun, Structure Analysis by Small-Angle
X-Ray and Neutron Scattering (Plenum Press, New York, 1987).

[19] R. Gähler, R. Golub, K. Habicht, T. Keller, and J. Felber, Physica B 229, 1 (1996).

[20] M. T. Rekveldt, J. Plomp, W. G. Bouwman, W. H. Kraan, S. Grigoriev, and M. Blaauw, Rev. Sci. Instrum. 76, 033901 (2005).

[21] M. T. Rekveldt, W. G. Bouwman, W. H. Kraan, O. Uca, S. Grigoriev, S. Habicht, and T. Keller, in Neutron Spin Echo, edited by F. Mezei, C. Pappas, and T. Gutberlet, Lecture Notes in Physics Vol. 601 (Springer, Berlin, 2003), pp. 87-99.

[22] P. Debye and A. M. Bueche, J. Appl. Phys. 20, 518 (1949).

[23] P. Debye, H. R. Andersson, and H. Brumberger, J. Appl. Phys. 28, 679 (1957).

[24] P. Z. Wong and Q. Z. Cao, Phys. Rev. B 45, 7627 (1992).

[25] M. Hunter, V. Backman, G. Popescu, M. Kalashnikov, C. W. Boone, A. Wax, V. Gopal, K. Badizadegan, G. D. Stoner, and M. S. Feld, Phys. Rev. Lett. 97, 138102 (2006).

[26] J. Török, S. Krishnamurthy, J. Kertész, and S. Roux, Phys. Rev. E 67, 021303 (2003).

[27] E. S. Kikkinides and V. N. Burganos, Phys. Rev. E 59, 7185 (1999).

[28] A. S. Balankin, O. Susarrey, and A. Bravo, Phys. Rev. E 64, 066131 (2001).

[29] M. Zaiser, F. M. Grassett, V. Koutsos, and E. C. Aifantis, Phys. Rev. Lett. 93, 195507 (2004).

[30] H. Hinrichsen and D. Wolf, The Physics of Granular Media (Wiley-VCH, Weinheim, 2004).

[31] R. Andersson, W. G. Bouwman, J. Plomp, F. M. Mulder, H. G. Schimmel, and I. M. de Schepper, (unpublished).

[32] www.degussa.com

[33] www.tekscan.com 